\title{
Evaluation of Osteoporosis in Hemophilic Arthropathy Patients: Correlation with Disease Severity and Serum Trace Minerals
}

\author{
Eiman Mahmoud Ghaniema, ${ }^{1}$ Sahar Fathi Ahmed, ${ }^{1}$ \\ Irene Raouf Amin, ${ }^{1}$ and Maryse Soliman Ayoub ${ }^{2}$ \\ ${ }^{1}$ Rheumatology and Rehabilitation Department, Ain Shams University, Cairo 11566, Egypt \\ ${ }^{2}$ Internal Medicine Department (Hematology Unit), Ain Shams University, Cairo 11566, Egypt \\ Correspondence should be addressed to Sahar Fathi Ahmed, saharfathi_283@yahoo.com
}

Received 27 November 2010; Revised 8 April 2011; Accepted 10 August 2011

Academic Editor: Pawel Szulc

Copyright (C) 2011 Eiman Mahmoud Ghaniema et al. This is an open access article distributed under the Creative Commons Attribution License, which permits unrestricted use, distribution, and reproduction in any medium, provided the original work is properly cited.

\begin{abstract}
Objective. To find out the presence of osteoporosis in hemophilic arthropathy patients and its correlation with clinical severity and serum levels of magnesium, copper, and zinc. Methods. Joint score, functional assessment score, bone densitometry, and serum magnesium, copper and zinc were done in twenty male hemophilic arthropathy patients and twenty controls. Results. There was highly significant lower $\mathrm{Z}$ scores of lumbar spine and neck of femur in patients versus controls $(P<0.011)$. $\mathrm{Z}$ score of neck of femur correlated negatively with total joint score $(P=0.013)$ and functional assessment score $(P=0.011)$. Serum levels of copper and zinc correlated positively with $\mathrm{Z}$ score of neck of femur $(P=0.004, P=0.001$, resp. $)$. Conclusion. Osteoporosis represents a frequent concomitant observation in hemophiliacs. Screening of young hemophiliacs for osteoporosis is recommended with measuring serum levels of magnesium, copper, and zinc for better management of the disease.
\end{abstract}

\section{Introduction}

Hemophilia is a coagulation disorder characterized by acute hemorrhages into the musculoskeletal system leading eventually to arthropathy and disability [1]. Deficiency of factor VIII (Hemophilia A) accounts for $85 \%$ of cases and $15 \%$ are due to factor IX deficiency (Hemophilia B). They are both inherited as $\mathrm{x}$-linked recessive disorder [2]. Hemoarthrosis occurs in $75 \%-90 \%$ of patients with hemophilia. The most common target joints are knee, ankle, and elbow [3].

Acute bleeding increases the pressure in the synovial cavity and bone marrow which leads to severe pain and possible osteonecrosis or a pseudotumoral mass. Intraarticular bleeding produces a direct chemical effect on synovium, cartilage, and bone. Overtime, the blood becomes deposited in the form of hemosiderin in these tissues. Recurrent hyperemia of the joint in the growing child causes juxta articular osteoporosis and overgrowth of the epiphysis. About $50 \%$ of patients with hemophilia develop permanent joint deformities [4].
Patients with severe hemophilia may be at risk for developing reduced bone density in childhood and adolescence for a number of reasons as arthropathy and joint deformities result in prolonged immobilization and reduced physical activity which predisposes them for osteoporosis. This can lead to increasing tendency of bone fragility and fractures in patients after trivial trauma [5].

Osteoporosis is a multifactorial disease with particular considerations to calcium, magnesium $(\mathrm{Mg})$, and other trace elements as copper $(\mathrm{Cu})$ and zinc $(\mathrm{Zn})$, as they are essential in bone metabolism as cofactors for specific enzymes for optimal bone matrix development and bone density sustenance [6].

Therefore, we aimed to assess the presence and severity of osteoporosis in patients with hemophilic arthropathy by determining dual energy X-ray absorptiometry (DEXA) and to correlate these findings with the extent of joint disease and serum levels of trace minerals as magnesium, copper, and zinc. 


\section{Patients and Methods}

Twenty male patients with hemophilia A were enrolled randomly for this study. Their age ranged from 7 to 40 years compared to 20 controls matched for age and sex. Those patients and controls were presenting to the Internal Medicine and Physical Medicine, Rheumatology, and Rehabilitation outpatient clinics of Ain Shams University Hospitals. The diagnosis of hemophilia was made clinically and useful to classify them according to measured factor VIII activity in the plasma as severe $<1 \mathrm{unit} / \mathrm{dL}$, moderate $1-5 \mathrm{unit} / \mathrm{dL}$, and mild $>5$ units/dL, according to Arnold and Hilgartner [7].

We excluded patients with cigarette smoking or alcohol abuse, patients with history of any chronic medical illness producing osteopenia/osteoporosis, thyroid or parathyroid disorders, history of chronic renal, hepatic, or gastrointestinal disease including parasitic infestations, patients with prolonged intake of steroids, antiepileptic medication, iron for anemia, Ca or Vit D supplementation, or any drug affecting bone metabolism.

All patients were subjected to the following.

(1) Joint Evaluation: Lower limb joints (ankles and knees) were assessed by using the clinical evaluation score of the world Federation of Hemophilia (WFH) which includes seven criteria and a total possible score of 12 [8]. Each joint was ascribed a score. Normal joints were scored as 0 , the highest possible score for knees and ankles was 48 . The sum of scores for both knees and ankles was used in the analysis [5].

(2) Juvenile Arthritis Functional Assessment Report (GAFAR): It is a single dimension scale developed by Howe et al. [9] based on 23 items used as a disability score to evaluate the functional status and daily living activities for each patient. The response to each activity was scored between 0 and 2 as follows: $0=$ indicating that the activity could be done alone without any difficulty during the previous week; $1=$ indicating that it could be done some of time and $2=$ indicating that it was almost never done alone. The score based on the 23 items was calculated as the sum of all items, assuming a range between 0 and 46 with lower score indicating better function.

(3) Radiological Evaluation:

(a) The roentgenographic examination included an anteroposterior projection and lateral views of both knee joints. The X-ray were classified according to Pettersson et al. [10] for standardized examination. The maximum possible score of a given joint was 13. The total score for both knee joints was 26.

(b) Bone densitometry: in all subjects, bone mineral density (BMD) was measured at the femoral neck and lumbar spine $\left(L_{1}-L_{4}\right)$ in the anterior and posterior projection using DEXA. Results were recorded for each patient as Z score (difference in SD from the mean of a healthy age- and gender-matched sample) [11].
(4) Laboratory Investigations:

(i) Hemoglobin for anemia.

(ii) Complete liver and kidney function tests.

(iii) Serological screening for HBs Ag and HCV.

(iv) Serum calcium $(\mathrm{Ca})$, phosphorus, and alkaline phosphatase to rule out osteomalacia and other metabolic bone disorders.

(v) Stool analysis to rule out any parasitic infestations.

(vi) Serum magnesium level was assayed using ADVIA 1650 (Payer, Siemens Healthcare Diagnostics) using modified xylidyl blue reaction, described by Mann and Yoe [12]. Reagent Code 74064. Normal range for serum is 1.9$2.5 \mathrm{mg} / \mathrm{dL}$.

(vii) Determination of copper and zinc in serum was done by using the flawless atomic absorption spectrometry (Perkin-Elmer Corp., Norwalk, Conn. Germany). $5 \mathrm{~mL}$ of blood was obtained after overnight fasting. Serum was diluted $1: 1$ with deionized water for copper and $1: 4$ with $1 \%$ glycerol for zinc determination [13, 14]. Results were expressed in $\mu \mathrm{g} / \mathrm{mL}$; normal levels for copper and zinc ranged from $0.7-1.0 \mu \mathrm{g} / \mathrm{mL}$ and $0.8-1.2 \mu \mathrm{g} / \mathrm{mL}$, respectively.

2.1. Statistical Methods. SPSS statistical software package (V. 18, IBM Corp., USA, 2010) was used for data analysis. Data were expressed as mean \pm SD for quantitative measures and both number and percentage for categorical data. Comparison between two independent groups of numerical parametric data was done using Student's $t$-test. Comparison between two independent groups of nonparametric data was done using Wilcoxon Rank Sum test. Ranked Spearman correlation test was done to study the possible association between each two variables among each group for nonparametric data. Probability of error at 0.05 was considered significant and highly significant at 0.001 .

\section{Results}

This study included 20 patients with hemophilic arthropathy and twenty healthy male subjects served as the control group. The patients' age ranged from 7 to 40 years, with a mean of $21.7 \pm 11.2$ years.

The disease duration ranged from 2 to 33 years with mean of $14.7 \pm 10.5$. The annual number of bleedings during the last 5 years ranged from 2 to 6 attacks per year with a mean of $4.45 \pm 1.43$. As regards therapy, we use cryoprecipitate and/or purified plasma derived factor eight, around 500-5000 units/kg/year according to requirements.

The plasma factor level (VIII) ranged from 210 units/dL, with a mean of $5.05 \pm 3.34$ (Table 1). According to the factor level, 8 patients (40\%) diagnosed as mild grade and 12 patients $(60 \%)$ diagnosed as moderate grade of hemophilia. Four patients (20\%) had positive hepatitis C virus. 
The number of clinically affected joints ranged from 1-4 (both knees and ankles). The total joint score (TJS) ranged from 4-21 with mean of $13.5 \pm 6.2$. Functional assessment score (JAFAR) ranged from $0-9$ with mean of $2.8 \pm 3.4$. The total X-ray score ranged from $6-19$ with a mean of $12.5 \pm 4.8$ (Table 1).

Total joint score correlated positively with total X-ray score $(r=0.58, P=0.006)$. In addition, both TJS and total X-ray score correlated positively with disease duration ( $r=0.46, P=0.03$ and $r=0.63, P=0.002$, resp. $)$, and correlated negatively with the factor level of hemophilia ( $r=-0.64, P=0.002$ and $r=-0.49, P=0.02$, resp.).

Functional assessment score correlated positively with TJS and total X-ray score $(r=0.81, P=0.001$ and $r=0.55$, $P=0.01$, resp.) and correlated negatively with factor level $(r=-48, P=0.03)$.

Presence of osteoporosis assessed by DEXA (Table 2) revealed highly significantly lower $Z$ scores of lumbar spine and neck of femur in hemophilic arthropathy patients versus controls $(P<0.001)$ (Table 2$)$. While, there was no significant difference in $\mathrm{Z}$ score of lumbar spine and neck of femur between patients with or without hepatitis $C$ virus $(P>0.05)$.

$\mathrm{Z}$ score of neck of femur correlated negatively with total joint score $(r=-0.547, P=0.01)$, functional assessment score $(r=-0.553, P=0.01)$, and total $\mathrm{X}$-ray score ( $r=-0.484, P=0.03)$. While, there was no significant correlation between $\mathrm{Z}$ score of lumbar spine and the clinical or radiological scores $(P>0.05)$.

In hemophilic arthropathy patients, a highly significant decrease was found in serum levels of $\mathrm{Mg}, \mathrm{Cu}$, and $\mathrm{Zn}$ compared to controls $(P<0.001)$, while there was no statistically significant difference as regards serum calcium levels $(P>0.05)$ (Table 3$)$.

Serum levels of $\mathrm{Zn}$ correlated negatively with TJS, functional assessment score, and total X-ray score $(r=$ $-0.51, P=0.01, r=-0.66, P=0.001$ and $r=-0.94$, $P=0.001$, resp.).

Serum levels of $\mathrm{Cu}$ and $\mathrm{Zn}$ correlated positively with $\mathrm{Z}$ score of neck of femur $(r=0.61, P=0.004$ and $r=0.83$, $P=0.001$, resp.) (Figures 1 and 2). On the other hand, there was no significant correlation between serum levels of either calcium or magnesium and the severity of osteoporosis as measured by $\mathrm{Z}$ score $(P>0.05)$.

\section{Discussion}

It has been suggested that the hemophiliacs may show a markedly lower bone mineral density (BMD) than the average population due to arthropathy that may induce lack of mobility as well as a pathological liver metabolism in those patients coinfected with a chronic hepatitis [15].

The possible role of functional interaction between hematopoietic and bone tissues in the development of age related osteoporosis is discussed by Gurevitch and Slavin [16]. Blood loss creating developmental pressure on hematopoietic system enhances production of hematopoietic growth factors and subsequently intensifies proliferation of
TABLE 1: Demographic, clinical, laboratory, and radiological data of hemophilic arthropathy patients.

\begin{tabular}{lcc}
\hline & \multicolumn{2}{c}{ Patients $(n=20)$} \\
& Range & Mean \pm SD \\
\hline Disease duration (years) & $2-33$ & $14.7 \pm 10.5$ \\
Factor level (units/dL) & $2-10$ & $5.05 \pm 3.3$ \\
Total joint score & $4-21$ & $13.5 \pm 6.2$ \\
Functional assessment score & $0-9$ & $2.8 \pm 3.4$ \\
Total X-ray score & $6-19$ & $12.5 \pm 4.8$ \\
\hline
\end{tabular}

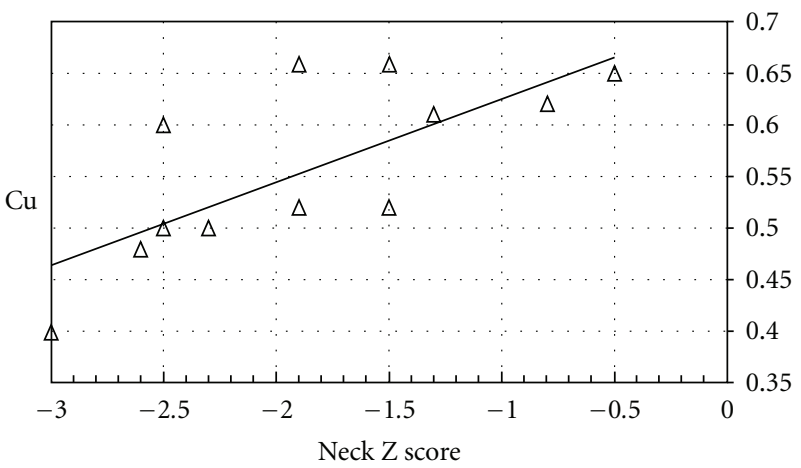

FIGURE 1: The positive correlation between serum cupper level and $\mathrm{Z}$ score of neck of femur among all patients.

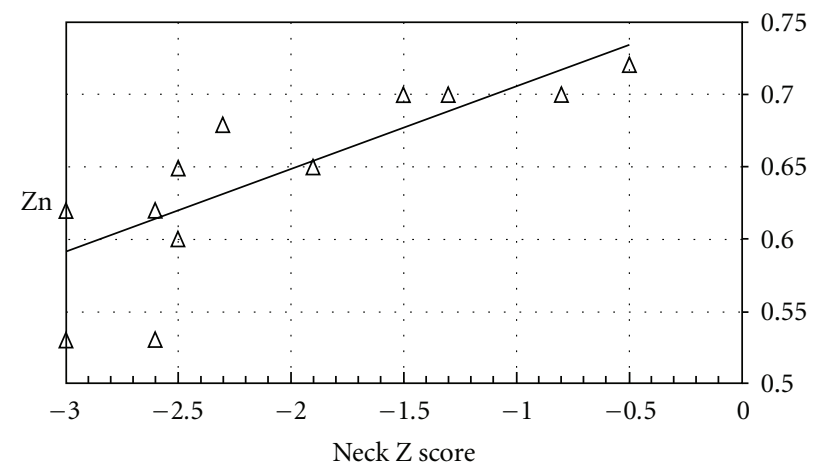

FIGURE 2: The positive correlation between serum zinc level and $\mathrm{Z}$ score of neck of femur among all patients.

hematopoietic progenitor cells, increasing the number of osteoclasts which intensifies resorption of bone tissue [17].

In addition, blood loss leads to the production of activity stimulating bone formation factors, extensive proliferation of osteogenic progenitor cells resulting in increased numbers of osteoblasts followed by new bone formation, and at same time increased production and maturation of osteoclasts which again enter the cycle of bone resorption together with exhaustion of osteogenic cell population for gradual development of osteoporosis [16]. Trace minerals may be important in maintaining bone quality through their role as metalloenzymes in the synthesis of collagen and other 
TABLE 2: DEXA results.

\begin{tabular}{|c|c|c|c|c|c|}
\hline & \multicolumn{2}{|c|}{ Patients $(n=20)$} & \multicolumn{2}{|c|}{ Controls $(n=20)$} & \multirow{2}{*}{$P$} \\
\hline & Range & Mean \pm SD & Range & Mean \pm SD & \\
\hline Z score lumbar & $-2.7-(-0.9)$ & $-2.1 \pm 0.6$ & $-0.2-1.3$ & $0.7 \pm 0.5$ & 0.001 \\
\hline Z score femur & $-3.0-(-0.5)$ & $-1.8 \pm 0.8$ & $-0.4-1.4$ & $0.7 \pm 0.6$ & 0.001 \\
\hline
\end{tabular}

TABle 3: Serum levels of Ca, Mg, Cu, and $\mathrm{Zn}$.

\begin{tabular}{|c|c|c|c|c|c|}
\hline & \multicolumn{2}{|c|}{ Patients $(n=20)$} & \multicolumn{2}{|c|}{ Controls $(n=20)$} & \multirow{2}{*}{$P$} \\
\hline & Range & Mean \pm SD & Range & Mean \pm SD & \\
\hline Calcium (mg/dL) & $9.1-9.6$ & $9.3 \pm 0.18$ & $9-9.5$ & $9.2 \pm 0.18$ & 0.26 \\
\hline Magnesium (mg/dL) & $1.5-1.8$ & $1.6 \pm 0.09$ & $1.9-2.5$ & $2.2 \pm 0.21$ & 0.001 \\
\hline Copper $(\mu \mathrm{g} / \mathrm{mL})$ & $0.40-0.66$ & $0.55 \pm 0.08$ & $0.7-1.0$ & $0.84 \pm 0.12$ & 0.001 \\
\hline $\operatorname{Zinc}(\mu \mathrm{g} / \mathrm{mL})$ & $0.53-0.72$ & $0.65 \pm 0.05$ & $0.8-1.2$ & $1.0 \pm 0.13$ & 0.001 \\
\hline
\end{tabular}

proteins that form the structure of bone [18]. They play important functional roles in bone metabolism and bone turnover. Zinc inhibits the differentiation of osteoclasts and promotes osteoblast activity affecting the formation of hard tissues. It could also increase bone growth factors and bone matrix protein, which are involved in the stimulation of bone formation and proliferation of osteoblastic cells [19].

Magnesium $(\mathrm{Mg})$ appears to be important in bone cell activity. It is shown to be mitogenic for osteoblasts, and its depletion causes cellular growth inhibition in vitro [20]. Copper $(\mathrm{Cu})$ is a cofactor for lysyl oxidase which is required in cross-linking of collagen and elastin. Cu deficiency causes inhibition of bone growth and osteoporosis as observed in Menkin's disease, an inherent inability to absorb $\mathrm{Cu}$ [21].

The present study aimed to find out the presence of osteoporosis in patients with hemophilic arthropathy and its correlation with clinical disease severity and serum levels of trace minerals as $\mathrm{Zn}, \mathrm{Cu}$ and $\mathrm{Mg}$.

In the present study, we used 3 scoring systems; clinical, functional and radiological scoring system to assess the extent of joint damage and related disability in 20 hemophilic patients. We scored only the lower limb major joints (knees and ankles), because these are the joints frequently affected by hemophilia, and damage of these joints will have a major impact on the patient's daily activities involving weight bearing. This was in agreement with, Gurcay et al. [1] who studied thirty one young patients with hemophilia aged between 3-18 years using the 3 scoring systems. They found that the most commonly affected joints in hemophilia are the hinge joints: knee, elbow, and ankles. Especially, the knees are most commonly affected in early childhood because of their weight bearing function.

$60 \%$ of our patients diagnosed as moderate grade of hemophilia, our results showed that the total joint score correlated positively with total X-ray score. In addition, both total joint and total X-ray scores correlated positively with disease duration and correlated negatively with serum factor level of hemophilia. This was in accordance with the study done by Gurcay et al. [1] who diagnosed 21 from 31 patients $(67.7 \%)$ as moderate grade and found that the clinical score correlated significantly with the radiological score. Their results were supported by those of Pettersson et al. [10] who concluded that Pettersson's score correlated very well with the clinical profile of patients with hemophilic arthropathy. In addition, they reported that total X-ray score increased with age. This may be primarily based on the duration of the disease and increased recurrent hemarthrotic attacks due to insufficient factor treatment.

In the current study, there was positive correlation between the JAFAR disability score, total joint score, and total $\mathrm{X}$-ray score. Dalyan et al. [22] reported a similar observation, as their analysis showed significant correlation between the disability and the radiological scores. Also, other study [1] found strong association between the disability score, radiological and clinical findings. The most difficult activities reported by our patients were walking 50 feet without help, standing up on tiptoes, and picking up something from the floor from a standing position. This was in accordance with Gurcay et al. [1] who concluded that these activities were limited mainly due to involvement of the joints of lower extremities which may easily result in functional disability.

In our study, presence of osteoporosis assessed by DEXA revealed highly significant lower $Z$ scores of lumbar spine and neck of femur in hemophilic arthropathy patients versus controls. This was in accordance with the study done by Wallny et al. [15] who found a relationship between hemophilia and osteoporosis and increased severity of hemophilia was associated with lower BMD in neck of femur. Also, Gallacher et al. [23] studied 19 patients with severe hemophilia A and showed significantly lower BMD values in comparison with controls.

In addition, $\mathrm{Z}$ score of neck of femur correlated negatively with total joint, functional assessment, and total $\mathrm{X}$ ray scores. This was in accordance with Barnes et al. [4] who found a statistically significant association between areal BMD Z scores and objective lower limb joint evaluation results. Patients with more established changes resulting from hemophilic joint disease exhibited the lowest BMD. Also, other studies [5, 15] found that patients with severe 
hemophilia are at risk of developing reduced BMD. Lastly, Khawaji et al. [24] stated that with increasing severity and number of affected joints, BMD significantly decreases.

There was no significant correlation between $\mathrm{Z}$ score of lumbar spine and either clinical or radiological scores. This was in accordance with Nair et al. [5] as the BMD of femoral neck showed over all better correlation with the examined variables than the BMD of the lumbar spine.

Similar observation was seen between our study and the previous studies done that no significant difference was found in $\mathrm{Z}$ scores of lumbar spine and neck of femur between patients with or without hepatitis $C$ virus.

In our study, we found a highly significant decrease in serum levels of $\mathrm{Mg}, \mathrm{Cu}$, and $\mathrm{Zn}$ among hemophilic patients compared to controls, while there was no statistically significant difference as regards serum Ca levels. Moreover, serum levels of $\mathrm{Cu}$ and $\mathrm{Zn}$ correlated positively with $\mathrm{Z}$ score of neck of femur and serum levels of $Z n$ correlated negatively with total joint, functional assessment, and total X-ray scores. On the other hand, there was no significant correlation between serum levels of either $\mathrm{Ca}$ or $\mathrm{Mg}$ and severity of osteoporosis as measured by Z score. Also, Mir et al. [19] found a significantly lower serum Zn concentration in men, with hip osteoporosis and concluded that zinc had a positive association with BMD in men and its deficiency was more common in osteoporotic

In addition, Mutlu et al. [6] found that serum $\mathrm{Mg}$ and Zinc were significantly lower in osteopenic women than in normal women. But no statistically significant differences were observed as regards copper levels. They also concluded that trace element supplementation especially with magnesium, zinc, and perhaps copper, may have beneficial effects on bone density. On the other hand, Gur et al. [18] stated that $\mathrm{Mg}, \mathrm{Cu}$, and $\mathrm{Zn}$ levels in serum of patients with postmenopausal osteoporosis were lower than those in controls demonstrating that deficiency of these trace minerals plays a major role in the development of osteoporosis when serum $\mathrm{Ca}$ and phosphorus were normal. Also, Odabasi et al. [21] reported significant differences between osteoporotic patients and controls as regards $\mathrm{Mg}$ concentration.

In conclusion, in hemophilic arthropathy patients, osteoporosis represents a frequent concomitant observation which may complicate the future treatment of these patients. Extensive joint disease is an important risk factor for the severity of osteoporosis. Trace minerals like $\mathrm{Mg}, \mathrm{Cu}$, and $\mathrm{Zn}$ are essential in bone metabolism as cofactors for specific enzymes that are essential for organic bone matrix synthesis. Screening of young hemophiliacs for reduced bone density is recommended with measuring the levels of $\mathrm{Mg}, \mathrm{Cu}$, and $\mathrm{Zn}$ for better assessment and management of the disease.

\section{Acknowledgments}

The authors would like to thank Dr. Yasser Ahmed Zaytoun, Professor of Clinical Pathology, Faculty of Medicine, Ain Shams University, for technical support.

\section{References}

[1] E. Gurcay, E. Eksioglu, U. Ezer, R. Tuncay, and A. Cakci, "Functional disability in children with hemophilic arthropathy," Rheumatology International, vol. 26, no. 11, pp. 10311035, 2006.

[2] E. C. Rodriguez-Merchan, "Orthopedic assessment in hemophilia," Hemophilia, vol. 9, supplement 1, pp. 65-74, 2003.

[3] R. Kashyap and V. P. Choudhry, "Management of hemophilia in developing countries," Indian Journal of Pediatrics, vol. 68, no. 2, pp. 151-157, 2001.

[4] C. Barnes, P. Wong, B. Egan et al., "Reduced bone density among children with severe hemophilia," Pediatrics, vol. 114, no. 2, pp. e177-181, 2004.

[5] A. P. Nair, F. Jijina, K. Ghosh, M. Madkaikar, M. Shrikhande, and M. Nema, "Osteoporosis in young haemophiliacs from Western India," American Journal of Hematology, vol. 82, no. 6, pp. 453-457, 2007.

[6] M. Mutlu, M. Argun, E. Kilic, R. Saraymen, and S. Yazar, "Magnesium, zinc and copper status in osteoporotic, osteopenic and normal post-menopausal women," Journal of International Medical Research, vol. 35, no. 5, pp. 692-695, 2007.

[7] W. D. Arnold and M. W. Hilgartner, "Hemophilic arthropathy. Current concepts of pathogenesis and management," Journal of Bone and Joint Surgery Series A, vol. 59, no. 3, pp. 287-305, 1977.

[8] M. Gilbert, "Prophylaxis: musculoskeletal evaluation," Seminars in Hematology, vol. 30, no. 2, pp. 3-6, 1993.

[9] S. Howe, J. Levinson, E. Shear et al., "Development of a disability measurement tool for juvenile rheumatoid arthritis. The Juvenile Arthritis Functional Assessment Report for children and their parents," Arthritis and Rheumatism, vol. 34, no. 7, pp. 873-880, 1991.

[10] H. Pettersson, A. Ahlberg, and I. M. Nilsson, "A radiologic classification of hemophilic arthropathy," Clinical Orthopaedics and Related Research, vol. 149, pp. 153-159, 1980.

[11] T. P. Millard, L. Antoniades, A. V. Evans, H. R. Smith, T. D. Spector, and J. N. W. N. Barker, "Bone mineral density of patients with chronic plaque psoriasis," Clinical and Experimental Dermatology, vol. 26, no. 5, pp. 446-448, 2001.

[12] C. K. Mann and J. H. Yoe, "Spectrophotometric determination of magnesium with 1-azo-2-hydroxy-3-(2.4-dimethylcarboxanilido)-naphtha- lene-1-(2-hydroxybenzene)," Analytica Chimica Acta, vol. 16, no. C, pp. 155-160, 1957.

[13] F. W. Sunderman and N. O. Roszel, "Measurements of copper in biologic materials by atomic absorption spectrometry," American Journal of Clinical Pathology, vol. 48, no. 3, pp. 286294, 1967.

[14] A. S. Prasad, D. Oberleas, and J. A. Halsted, "Determination of zinc in biological fluids by atomic absorption spectrophotometry in normal and cirrhotic subjects," The Journal of Laboratory and Clinical Medicine, vol. 66, no. 3, pp. 508-516, 1965.

[15] T. A. Wallny, D. T. Scholz, J. Oldenburg, and C. Nicolay, "Osteoporosis in hemophilia-an underestimated co morbidity?” Hemophilia, vol. 13, no. 1, pp. 79-84, 2007.

[16] O. Gurevitch and S. Slavin, "The hematological etiology of osteoporosis," Medical Hypotheses, vol. 67, no. 4, pp. 729-735, 2006.

[17] D. Visnjic, Z. Kalajzic, D. W. Rowe, V. Katavic, J. Lorenzo, and H. L. Aguila, "Hematopoiesis is severely altered in mice with 
an induced osteoblast deficiency," Blood, vol. 103, no. 9, pp. 3258-3264, 2004.

[18] A. Gur, L. Colpan, and K. Nas, "The role of trace minerals in the pathogenesis of post-menopausal osteoporosis and a new effect of calcitonin," Journal of Bone and Mineral Research, vol. 20, pp. 39-43, 2002.

[19] E. Mir, A. Hossein-Nezhad, A. Bahrami et al., "Serum zinc concentration could predict bone mineral density and protect osteoporosis in healthy men," Iranian Journal of Public Health, vol. 36, pp. 30-36, 2007.

[20] R. K. Rude and H. E. Gruber, "Magnesium deficiency and osteoporosis: animal and human observations," Journal of Nutritional Biochemistry, vol. 15, no. 12, pp. 710-716, 2004.

[21] E. Odabasi, M. Turan, A. Aydin, C. Akay, and M. Kutlu, "Magnesium, zinc, copper, manganese, and selenium levels in postmenopausal women with osteoporosis. Can magnesium play a key role in osteoporosis?" Annals of the Academy of Medicine Singapore, vol. 37, no. 7, pp. 564-567, 2008.

[22] M. Dalyan, S. Tuncer, and S. Kemahli, "Hemophilic arthropathy: evaluation of clinical and radiological characteristics and disability," Turkish Journal of Pediatrics, vol. 42, no. 3, pp. 205209, 2000.

[23] S. J. Gallacher, C. Deighan, A. M. Wallace et al., "Association of severe haemophilia A with osteoporosis: a densitometric and biochemical study," Quarterly Journal of Medicine, vol. 87, no. 3, pp. 181-186, 1994.

[24] M. Khawaji, K. Akesson, and E. Berntorp, "Long-term prophylaxis in severe hemophilia seems to preserve bone mineral density," Hemophilia, vol. 15, pp. 261-266, 2009. 


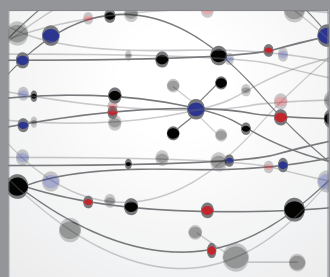

The Scientific World Journal
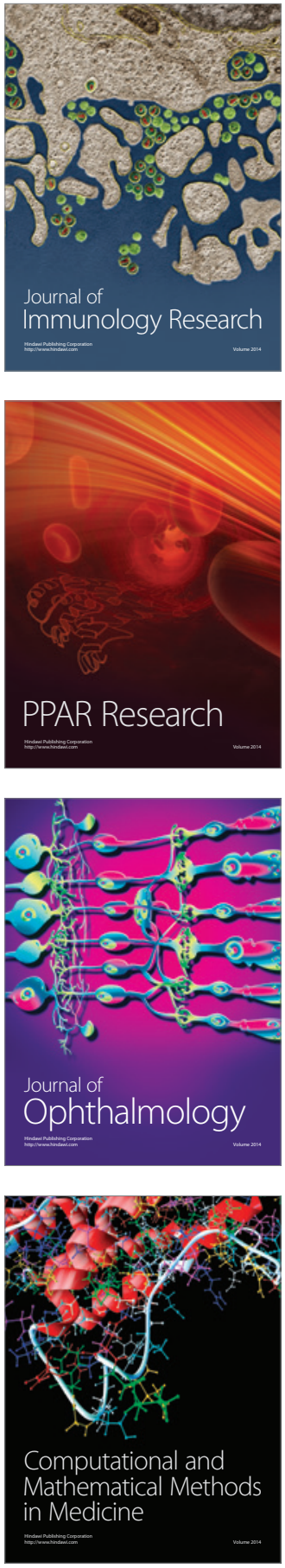

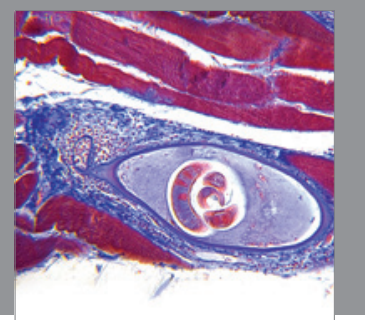

Gastroenterology

Research and Practice
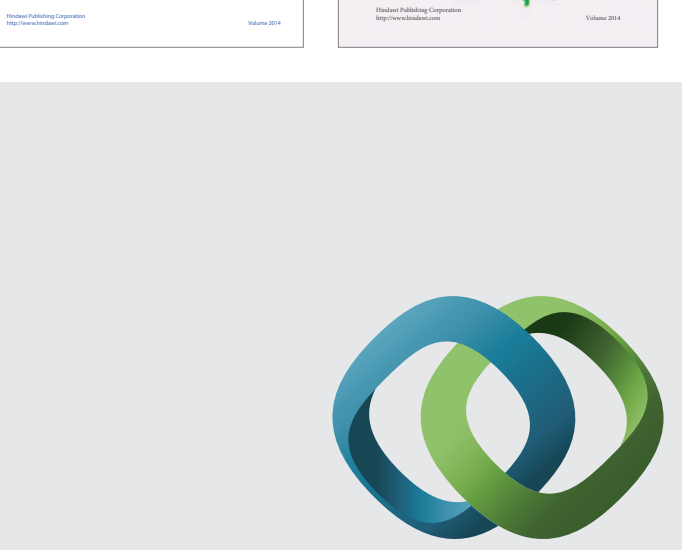

\section{Hindawi}

Submit your manuscripts at

http://www.hindawi.com
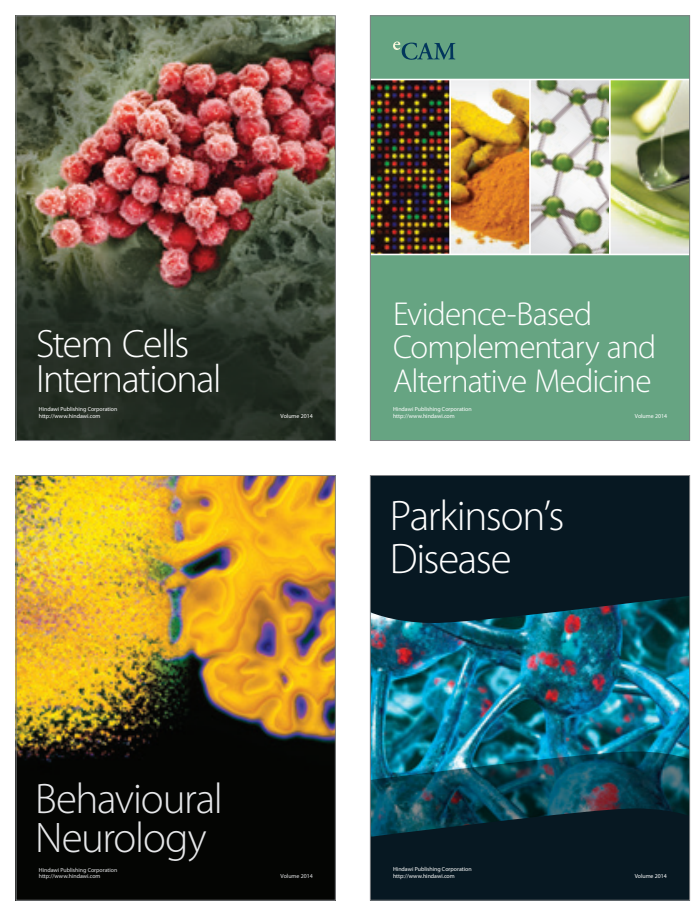

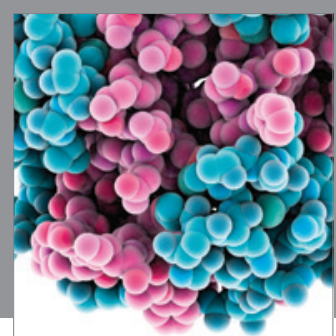

Journal of
Diabetes Research

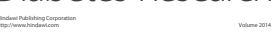

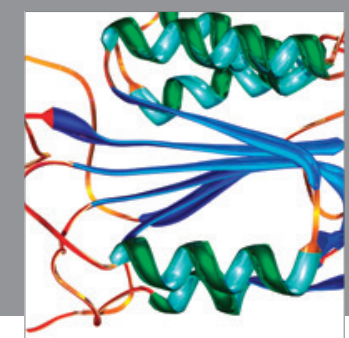

Disease Markers
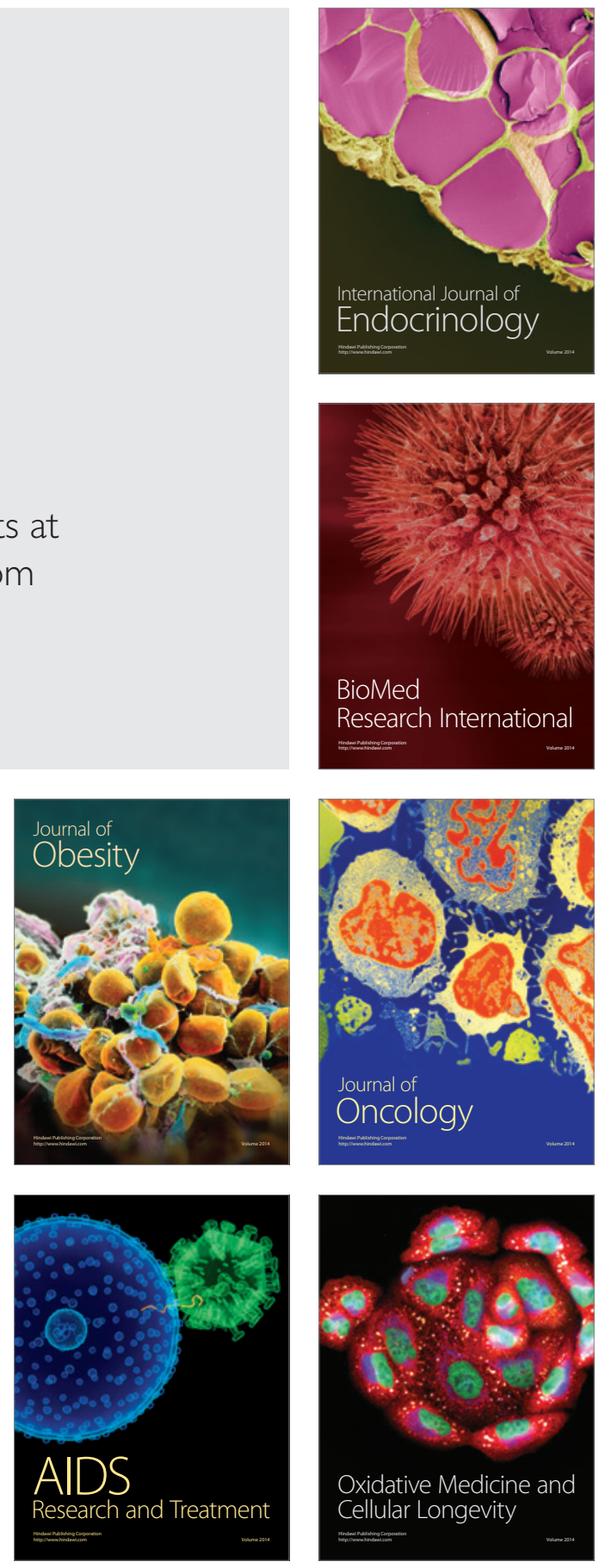